\title{
Three-dimensional tumor spheroids for in vitro analysis of bacteria as gene delivery vectors in tumor therapy
}

\author{
Annika Osswald ${ }^{1,4 \dagger}$, Zhongke Sun ${ }^{1,2 \dagger}$, Verena Grimm ${ }^{1}$, Grace Ampem¹, Karin Riegel ${ }^{1}$, Astrid M. Westendorf ${ }^{3}$, \\ Wolfgang Sommergruber ${ }^{4}$, Kerstin Otte ${ }^{5}$, Peter Dürre ${ }^{1}$ and Christian U. Riedel ${ }^{1^{*}}$
}

\begin{abstract}
Background: Several studies in animal models demonstrated that obligate and facultative anaerobic bacteria of the genera Bifidobacterium, Salmonella, or Clostridium specifically colonize solid tumors. Consequently, these and other bacteria are discussed as live vectors to deliver therapeutic genes to inhibit tumor growth. Therapeutic approaches for cancer treatment using anaerobic bacteria have been investigated in different mouse models. In the present study, solid three-dimensional (3D) multicellular tumor spheroids (MCTS) of the colorectal adenocarcinoma cell line HT-29 were generated and tested for their potential to study prodrug-converting enzyme therapies using bacterial vectors in vitro.
\end{abstract}

Results: HT-29 MCTS resembled solid tumors displaying all relevant features with an outer zone of proliferating cells and hypoxic and apoptotic regions in the core. Upon incubation with HT-29 MCTS, Bifidobacterium bifidum S17 and Salmonella typhimurium YB1 selectively localized, survived and replicated in hypoxic areas inside MCTS. Furthermore, spores of the obligate anaerobe Clostridium sporogenes germinated in these hypoxic areas. To further evaluate the potential of MCTS to investigate therapeutic approaches using bacteria as gene delivery vectors, recombinant bifidobacteria expressing prodrug-converting enzymes were used. Expression of a secreted cytosine deaminase in combination with 5-fluorocytosine had no effect on growth of MCTS due to an intrinsic resistance of HT-29 cells to 5-fluorouracil, i.e. the converted drug. However, a combination of the prodrug CB1954 and a strain expressing a secreted chromate reductase effectively inhibited MCTS growth.

Conclusions: Collectively, the presented results indicate that MCTS are a suitable and reliable model to investigate live bacteria as gene delivery vectors for cancer therapy in vitro.

Keywords: Bacteria, Gene delivery vector, Tumor targeting, Prodrug converting enzyme, Tumor spheroids

\section{Background}

Solid tumors are characterized by hypoxic and necrotic areas, which are the result of high metabolic activity of tumor tissue and concomitant lack of oxygen supply due to insufficient vasculature [1, 2]. Hypoxic areas of solid tumors have an increased resistance to chemotherapy and radiation compared to better oxygenated tumor

\footnotetext{
*Correspondence: christian.riedel@uni-ulm.de

${ }^{\dagger}$ Annika Osswald and Zhongke Sun have contributed equally to this work

${ }^{1}$ Institute of Microbiology and Biotechnology, University of Ulm,

89069 Ulm, Germany

Full list of author information is available at the end of the article
}

tissue [3-7]. This has fuelled the search for alternative or supplementary therapeutic strategies. One promising approach is the use of bacterial vectors for expression of therapeutic genes directly in tumor tissue. Different bacterial species including Escherichia coli $[8,9]$ and Listeria monocytogenes $[10,11]$ were shown to selectively colonize tumors in various animal models. The most frequently investigated bacterial gene delivery systems to target solid tumors are, however, facultative or obligate anaerobes of the genera Salmonella, Clostridium, and Bifidobacterium (reviewed in [12-15]). 
Bifidobacteria are Gram-positive, obligate anaerobic bacteria of the normal human intestinal microbiota. Several studies in animal models have shown that bifidobacteria selectively colonize and replicate in solid tumors following oral, intravenous, or intratumoral application $[16,17]$. Due to their non-pathogenic nature and broad use as probiotics, bifidobacteria are promising candidates as live vectors for delivery and expression of therapeutic genes to inhibit tumor growth. However, most bifidobacteria are highly resistant to genetic manipulation and only a very limited number of strains have been modified to express genes relevant for tumor therapy [18-20].

Another group of Gram-positive, strictly anaerobic bacteria widely used for tumor targeting strategies are spore-forming Clostridium sp. [21, 22]. In early studies, clostridia were mainly used as tumor therapeutics based on their oncolytic activity (reviewed in [14, 22]). However, their oncolytic effect was limited to large tumors with extensive and strictly hypoxic areas, tumor regression was incomplete and experimental animals died from clostridial toxins [14]. Consequently, this problem was addressed by generation of attenuated or non-pathogenic strains [23-25]. A major advantage of clostridia is that these microorganisms are able to form spores, which are immunologically inert and can be administered safely by intravenous injection [22, 26]. Spores of various Clostridium sp. were shown to selectively germinate in tumors and vegetative cells are non-viable in other, more oxygenated tissues [22]. These features make spores an attractive alternative for administration of bacterial gene vectors to tumor patients. A clinical phase I safety study utilizing Clostridium novyi-NT spores has been performed in patients with various treatment-refractory solid tumor malignancies [27]. The non-toxic C. novyi-NT strain was generated by inactivation of the phage carrying the lethal C. novyi toxin [25]. In animal experiments, a single intravenous injection of $C$. novyi-NT spores resulted in efficient colonization of tumors and caused tumor regression. Moreover, tumors did not recur in approximately $30 \%$ of the animals $[25,28]$.

Similarly, facultative anaerobic Salmonella sp. were shown to colonize solid tumors [29, 30]. Various recombinant Salmonella typhimurium strains have been generated for different therapeutic strategies, e.g. expression of PCEs, immunomodulatory molecules or bacterial toxins (reviewed in [29, 30]). Additionally, several attempts were made to improve tumor colonization of S. typhimurium and to reduce effects of this human pathogen on normal tissues [31]. For example, deletion of $m s b B$, whose gene product is involved in myristoylation of lipid A, results in attenuated virulence and a reduced inflammatory response [32]. Tumor-specificity was improved by generation of mutant strains with metabolic defects that can be complemented by nutrients specifically available in tumors. To this end, genes such as aroA and purI, which encode for crucial steps in purine and amino acid biosynthesis, were targets for the generation of auxotrophic mutants of S. typhimurium [33-35].

Based on the attenuated vaccine strain S. typhimurium SL7207, which carries an inactivated aroA gene [33], the "obligate" anaerobic S. typhimurium YB1 was generated [36]. S. typhimurium YB1 expresses an RNA molecule complementary to the asd mRNA exclusively under aerobic conditions. This silences asd expression by an anti-sense RNA mechanism during aerobic growth. The asd gene encodes for an enzyme required for biosynthesis of diaminopimelic acid (DAP), which is an essential component of the bacterial cell wall. In consequence, $S$. typhimurium YB1 is auxotrophic for DAP under aerobic conditions but is able to grow in the absence of DAP under anaerobic conditions when the anti-sense RNA is not expressed. The result is an attenuated strain that shows improved colonization and survival in hypoxic areas of tumors but not in other, more oxygenated organs of mice [36].

A widely used in vitro model system in preclinical cancer research are three-dimensional (3D) spherical cell aggregates, so-called multicellular tumor spheroids (MCTS), which are formed by a wide range of tumor cell lines when cultured under appropriate conditions [37, 38]. These MCTS display characteristic features of solid tumors including different zones of proliferating, apoptotic, and necrotic cells and an oxygen gradient with hypoxic areas in the core [3, 37-39]. Besides the physiological parameters, MCTS also more closely resemble the global expression profiles of tumor biopsies than classical 2D cell cultures $[40,41]$. Based on these properties, MCTS grown in vitro are increasingly used for drug discovery and pharmacokinetic and pharmacodynamic studies [37-39, 42].

The objective of the presented study was to evaluate the potential of MCTS as an in vitro model system for preclinical investigation of therapeutic strategies to treat cancer using anaerobic bacteria as gene delivery vectors.

\section{Results}

\section{Characterization of MCTS of the colorectal} adenocarcinoma cell line HT-29

Previous studies have shown that different bacteria are able to selectively colonize subcutaneous tumors in mice $[16,17]$. In order to investigate bacterial tumor targeting in vitro, MCTS of the colorectal cancer cell line HT-29 were established as a 3D model for solid tumors. For the generation of MCTS, HT-29 cells were grown in ultralow attachment cell culture plates. Over a 14-day period, MCTS steadily increased in size (data not shown) and 
reached a diameter of 500-1000 $\mu \mathrm{m}$. HT-29 MCTS displayed an intense purple hematoxylin staining in the outer layers of the MCTS, which gradually fainted into a pink eosin staining towards the core (Additional file 1: Figure S1A). The inner core of MCTS completely lacked fixable structures. Moreover, HT-29 MCTS were characterized by hypoxic areas inside MCTS starting approximately $100 \mu \mathrm{m}$ below the outer surface and apoptotic cells appeared another 10-20 $\mu \mathrm{m}$ further towards the core (Additional file 1: Figure S1B). Proliferating tumor cells were observed exclusively on the outermost $100 \mu \mathrm{m}$ in the area where actin staining indicates an intact cytoskeleton (Additional file 1: Figure S1C).

\section{Localization and survival of B. bifidum S17 in MCTS}

Since MCTS display hypoxic areas similar to those observed in tumors in vivo, the ability of obligate anaerobic bifidobacteria to colonize MCTS in vitro was tested.
B. bifidum S17 or fluorescent derivatives were incubated with MCTS for $48 \mathrm{~h}$. In cryosections of these MCTS, B. bifidum S17 localized predominantly in the core of MCTS and this signal co-localized with hypoxic areas (Fig. 1a) and the necrotic regions of MCTS inside the apoptotic areas (Fig. 1b). In higher magnification images, individual, rod-shaped, red-fluorescent B. bifidum S17/ pVG-mCherry could be visualized around Hoechststained cell nuclei indicating a close association of bacteria with tumor cells (Fig. 1c).

In order to check if B. bifidum S17 is able to reach the core of MCTS in a viable state and survive in this niche for prolonged periods, experiments were repeated and viable bacteria inside MCTS were quantified for up to $72 \mathrm{~h}$ following infection. Indeed, the number of viable B. bifidum S17 remained more or less constant throughout the experiment (Fig. 1d). By contrast, in plain culture media, i.e. in the absence of MCTS, viable B. bifidum a

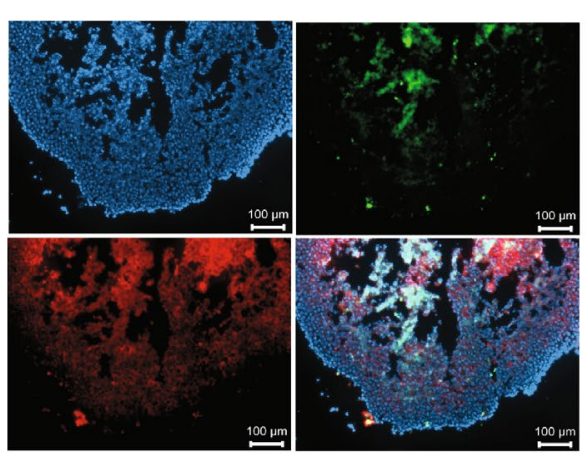

Hoechst / S17 / Hypoxia

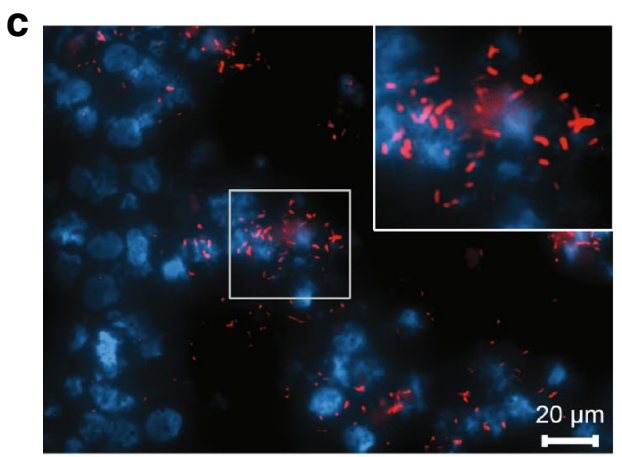

Hoechst / S17

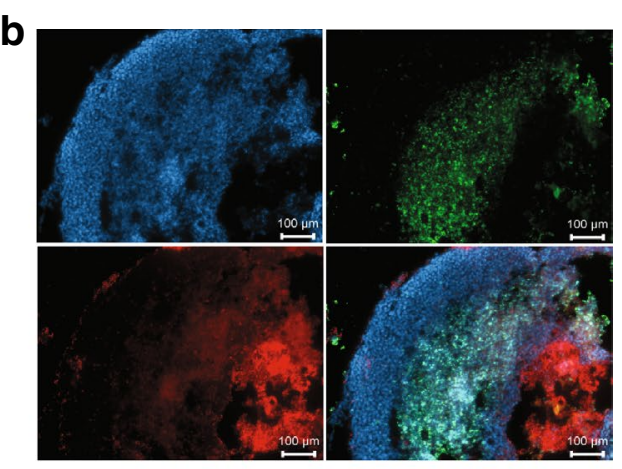

Hoechst / Apoptosis / S17

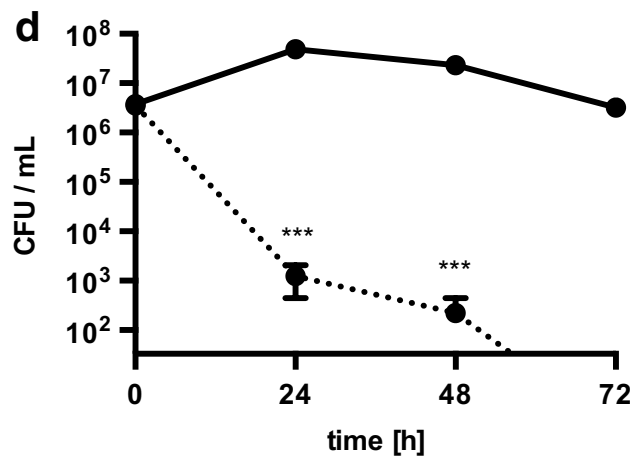

MCTS $\bullet \cdot$ medium

Fig. 1 Localization and survival of B. bifidum S17 in MCTS. HT-29 MCTS were incubated with B. bifidum S17/pVG-GFP (a S17, green) or B. bifidum S17/ pVG-mCherry (b, c S17, red) for 48 h. Cryosections of MCTS were stained for hypoxia (a Hypoxyprobe-Red549, red) or apoptosis (b TUNEL, green). Nuclei were stained with Hoechst. Images were acquired with a $\times 10$ (in $\mathbf{a}, \mathbf{b}$ scale bars $100 \mu$ m; all three fluorescence channels are shown plus the merged image in the lower right panel) or $\times 40$ (in c scale bar $20 \mu \mathrm{m}$ ) objective. The inset in the upper right corner of $\mathbf{c}$ is a digital zoom on the image part marked with a white box. d Viable bacteria of B. bifidum S17 WT in HT-29 MCTS were determined at different time points after inoculation. As controls (medium), bacteria were incubated under identical conditions in the absence of MCTS. For each strain, time-point and condition, six tumors were infected and combined for preparation of tumor lysates. Values are colony-forming units (CFU) per mL lysate and are mean \pm standard error of the mean (SEM) of three tumor lysates infected with independent bacterial cultures. Statistical analysis was performed for each time-point using Student's $t$ test $\left({ }^{* *} P<0.001\right)$ 
S17 counts dropped by more than 4 orders of magnitude within the first $24 \mathrm{~h}$ and were below the detection limit after $72 \mathrm{~h}$. Control experiments were performed by incubation of B. bifidum S17 with monolayers of HT-29 cells. As observed for plain culture media, viable counts dropped below the detection limit within $72 \mathrm{~h}$ (data not shown) demonstrating that presence of HT-29 cells alone is not sufficient but instead formation of MCTS is required to maintain viability.

\section{Colonization of HT-29 MCTS by other tumor targeting bacteria}

Salmonella sp. are the most frequently used bacteria in tumor targeting studies. One example is S. typhimurium $\mathrm{YB} 1$, a strain that was rendered obligate anaerobic to improve selective localization and survival in hypoxic tumor tissue [36]. Following co-incubation, S. typhimurium YB1 was mainly present in the core of MCTS although some bacteria were also detected on the outer surface of the MCTS (Fig. 2a). Moreover, bacteria were present mainly in areas that also stained positive for hypoxia and apoptosis (Fig. 2a-c). Experiments to quantify viable bacteria in MCTS showed that $S$. typhimurium YB1 is able to survive inside tumor spheroids for at least $72 \mathrm{~h}$ (Fig. 2d). In fact, after a slight decrease during the first $24 \mathrm{~h}$, viable $S$. typhimurium YB1 increased tenfold over the next $48 \mathrm{~h}$ indicating replication of S. typhimurium YB1 in MCTS. Over the same period, the number of viable bacteria in controls (i.e. bacteria in medium without MCTS) decreased by about 4 orders of magnitude.

Another group of bacteria frequently used as gene delivery vector in cancer therapy are spore-forming a

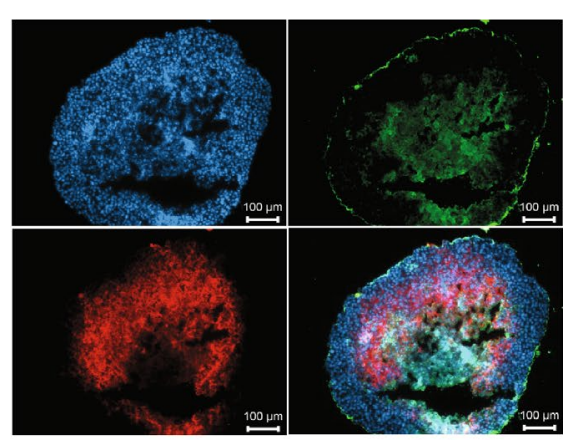

Hoechst / YB1 / Hypoxia

C



b



Hoechst / apoptosis / YB1

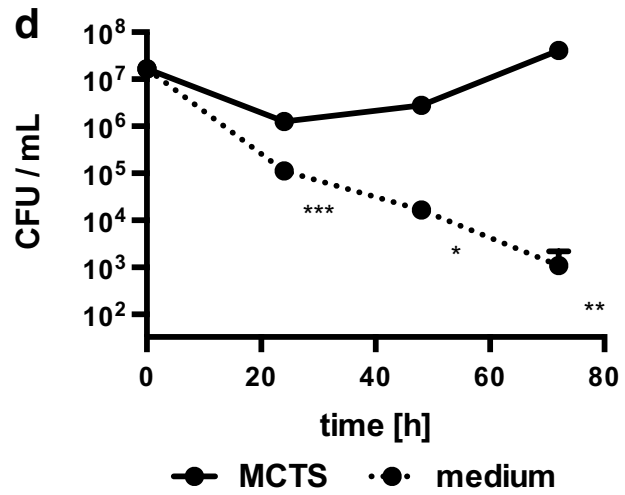

Fig. 2 Localization and survival of S. typhimurium YB1 in MCTS. HT-29 MCTS were incubated with S. typhimurium YB1 (YB1, green). After 48 h, MCTS cryosections were stained for S. typhimurium (a, c Alexa Fluor ${ }^{\circledR} 488$-conjugated secondary antibody, green; b Alexa Fluor ${ }^{\circledR} 555$-conjugated secondary antibody, red) and hypoxia (a, c Hypoxyprobe, red) or apoptosis (b TUNEL, green). Nuclei were stained with Hoechst (blue). Images were acquired with a $\times 10$ (a, b scale bars $100 \mu \mathrm{m}$; all three fluorescence channels are shown plus the merged image in the lower right panel) or $\times 100$ (in $\mathbf{c}$ scale bar $10 \mu \mathrm{m}$ ) objective. The image in $\mathbf{c}$ is acquired at a location inside the hypoxic area of an MCTS. The inset in the upper right corner of $\mathbf{c}$ is a digital zoom on the image part marked with a white box. d Viable bacteria of S. typhimurium YB1 in HT-29 MCTS at different time points after inoculation. As controls (medium), bacteria were incubated under identical conditions in the absence of MCTS. For each strain, time-point and condition, six tumours were infected and combined for preparation of tumour lysates. Values are colony-forming units (CFU) per mL lysate and are mean \pm SEM of three tumour lysates infected with independent bacterial cultures (in some cases, error bars are smaller that graph symbols). Statistical analysis was performed using Student's $t$ test $\left({ }^{*} P<0.05 ;{ }^{* *} P<0.01 ;{ }^{* * *} P<0.001\right)$ 
clostridia [22]. In order to test the utility of MCTS to study tumor targeting using clostridial spores, MCTS were incubated for 7 days with a pure spore preparation of C. sporogenes NCIMB 10696. Clostridial spores accumulated exclusively inside MCTS starting about $100 \mu \mathrm{m}$ underneath the outer surface (Fig. 3a), which correlates with the beginning of hypoxia (Additional file 1: Figure S1B). Upon closer examination, structures that stained positive for Hoechst were observed in areas of stained spores (Fig. 3b). These structures clearly differed morphologically from cell nuclei and had the size and shape of bacilli, i.e. the vegetative form of C. sporogenes (Fig. 3c) suggesting germination of spores.

\section{Generation of HT-29/GFP MCTS}

In order to test potential therapeutic strategies using bacteria as gene delivery vectors, fluorescent MCTS were generated. For this purpose, HT-29 cells were stably transfected with pEGFP-N3 (Clontech Laboratories, USA; Additional file 2: Materials S2). HT-29/EGFP4, a clone exhibiting stable, bright fluorescence, absence of EGFP-negative cells (Additional file 3: Figure S2A) and identical growth characteristics as the parental cell line (data not shown), was selected for further experiments. After 14 days of incubation, MCTS of HT-29/EGFP4 cells had the same size as those of the parental cell line but showed a uniform green fluorescent label throughout the MCTS (Additional file 3: Figure S2B). To test if strategies to inhibit growth of MCTS can be monitored using this system, mature HT-29/EGFP4 MCTS were treated with staurosporine. Fluorescence intensity of untreated HT-29/EGFP4 MCTS increased by $70-80 \%$ over a $96 \mathrm{~h}$ period. By contrast, HT-29/EGFP4 MCTS treated with 30 or $300 \mathrm{nM}$ staurosporine showed no increase in EGFP fluorescence (Additional file 3: Figure S2C) indicating efficient inhibition of MCTS growth. This suggests that HT-29/EGFP4 MCTS are a valid model to study therapeutic approaches to inhibit tumor growth.

\section{Evaluation of PCE strategies in HT-29/GFP MCTS}

In a previous study, we generated B. bifidum S17/pAOSO_CD, a strain expressing a secreted derivative of the E. coli cytosine deaminase [43]. This bacterial enzyme is not present in mammalian cells and converts the nontoxic prodrug 5-FC to the potent cytostatic compound 5-FU, which inhibits DNA synthesis. The potential of B. bifidum S17/pAO-S0_CD in combination with 5-FC to inhibit tumor growth in vitro was analyzed using HT-29/EGFP4 MCTS (Fig. 4a). Fluorescence of untreated MCTS increased by approx. $60 \%$ over the $96 \mathrm{~h}$ monitored and staurosporine-treated control MCTS showed significantly decreased EGFP intensity. Neither B. bifidum S17/pAO-S0_CD, nor 5-FC, or the combination of
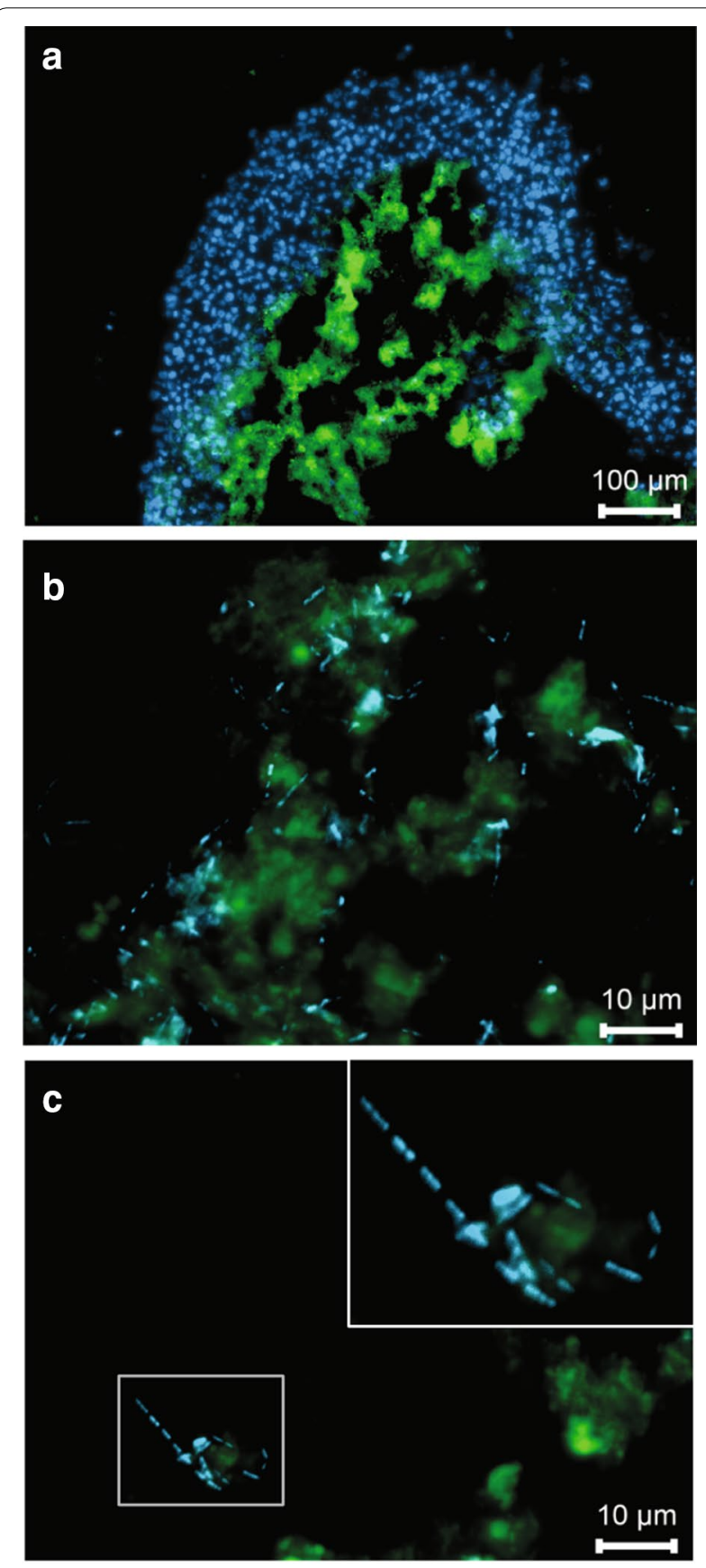

Hoechst / spores

Fig. 3 Germination of C. sporogenes spores in MCTS. HT-29 MCTS were infected with $1 \times 10^{3}$ spores and incubated for 7 days. Cryosections of MCTS were then stained for spores (FITC-conjugated antiserum, green). Nuclei and DNA of vegetative bacterial cells were stained with Hoechst (blue). Images were acquired with a $\times 10$ (in a scale bar $100 \mu \mathrm{m}$ ), or $\times 100$ (in b, c scale bars $10 \mu \mathrm{m}$ ) objective. Higher magnification images in $\mathbf{b}, \mathbf{c}$ are acquired at locations inside the hypoxic area of MCTS. The inset in the upper right corner of $\mathbf{c}$ is a digital zoom on the image part marked with a white box. Images are composite of both fluorescence channels and one representative image of three independent experiments is shown 



Fig. 4 Effect of B. bifidum S17/pAO-SO_CD and 5-FC on HT-29/EGFP4 MCTS. a HT-29/EGFP4 MCTS were incubated with B. bifidum S17/ PAO-S0_CD (S17 CD) alone or in combination with $0.25 \mathrm{mg} / \mathrm{mL} 5-\mathrm{FC}$ up to for $96 \mathrm{~h}$. As controls, MCTS were treated with $200 \mathrm{nM}$ staurosporine (stauro), 5-FC, or medium alone (-). b HT-29/EGFP4 MCTS were grown in the presence and absence of 0.5 or $0.05 \mathrm{mg} / \mathrm{mL} 5-\mathrm{FU}$. All MCTS were analyzed for growth by fluorescence measurements for $96 \mathrm{~h}$. Fluorescence intensities were normalized to give $100 \%$ for each MCTS at the start of the experiment $(t=0 \mathrm{~h})$. Values are relative fluorescent units (RFU) per MCTS and are mean \pm SEM of three MCTS Results of one representative of three independent experiments are shown. Statistical analysis was performed using two-way ANOVA with Bonferroni post-test correction for multiple comparisons (***difference to untreated MCTS significant with adjusted $P<0.001$, all other comparisons not significant)

both, resulted in a reduction of the fluorescence intensity below control levels.

One possible explanation for the lack of an effect on MCTS by the combination of B. bifidum S17/pAOSO_CD and 5-FC could be an intrinsic resistance of the HT-29 cell line to 5-FU treatment, as reported for different colon cancer cells $[44,45]$. To test this hypothesis, the pure compound 5-FU was added to HT29/EGFP4 MCTS. Interestingly, none of the tested concentrations of 5-FU was able to inhibit the increase in EGFP intensity during MCTS growth, indicating that the clone of HT-29 cells used to generate MCTS was resistant to 5-FU (Fig. 4b).

To test a second PCE strategy, pMgapS6C (Fig. 5a) was generated and transformed into B. bifidum S17. The recombinant strain B. bifidum S17/pMgapS6C expresses a secreted chromate reductase. This enzyme belongs to the family of nitroreductases and converts the prodrug CB1954 to hydroxylamines, which intercalate into DNA and efficiently inhibit tumor cell growth [46]. Culture supernatants of B. bifidum S17/pMgapS6C contained highly increased nitroreductase activity compared to the control strain (Fig. 5b).

Incubation with B. bifidum S17/pMgapS6C and CB1954 resulted in efficient growth inhibition of HT-29/EGFP4 MCTS compared to the negative controls. The EGFP signal of MCTS treated with B. bifidum S17/pMgapS6C or CB1954 alone did not differ from untreated controls. However, fluorescence of MCTS treated with a combination with B. bifidum S17/pMgapS6C and CB1954 was reduced significantly and was comparable to that observed with staurosporine treatment (Fig. 5c). This indicates efficient growth inhibition of HT-29/EGFP4 MCTS upon treatment with the recombinant strain and the prodrug.

\section{Discussion}

Delivery of therapeutic genes by anaerobic bacteria is an attractive alternative or supplementary strategy in cancer treatment [12-15]. The vast majority of studies on bacterial tumor targeting have been performed in mouse models. In the present study, 3D MCTS of the colorectal adenocarcinoma cell line HT-29 were evaluated as a model system to investigate bacterial tumor targeting in vitro.

HT-29 MCTS displayed (patho)physiologically relevant features of solid tumors. Size and organization of these MCTS into a proliferating outer zone and increasing hypoxia, apoptosis and necrosis towards the core are in line with previous reports [37,38]. The presented data provides direct evidence for a selective localization of tumor targeting bacteria in hypoxic areas of 3D tumor spheroids. B. bifidum S17 derivatives expressing the fluorescent proteins GFP or mCherry were detected almost exclusively in hypoxic areas of MCTS. S. typhimurium YB1 localized preferentially to hypoxic areas of MCTS and appeared to proliferate in this niche (Fig. 2). Both strains are able to survive at high rates for at least $72 \mathrm{~h}$ in MCTS. By contrast, viable bacteria of both strains decreased significantly in controls, i.e. medium without MCTS. Slight differences in the viability under these conditions were noted with a more rapid decrease observed for B. bifidum S17 compared to S. typhimurium YB1. This may be due to the fact that bifidobacteria are obligate anaerobes to which presence of oxygen is detrimental. S. typhimurium strains are facultative anaerobes, which are able to grow without oxygen but prefer growth under aerobic conditions. The YB1 strain is genetically modified in a way that they are not able to grow in the presence of oxygen [36]. However, oxygen is not toxic to YB1 which maybe the reason for the better survival of YB1 under cell culture conditions.

Bifidobacterium bifidum S17 and S. typhimurium YB1 were found in slightly different locations of the MCTS. 

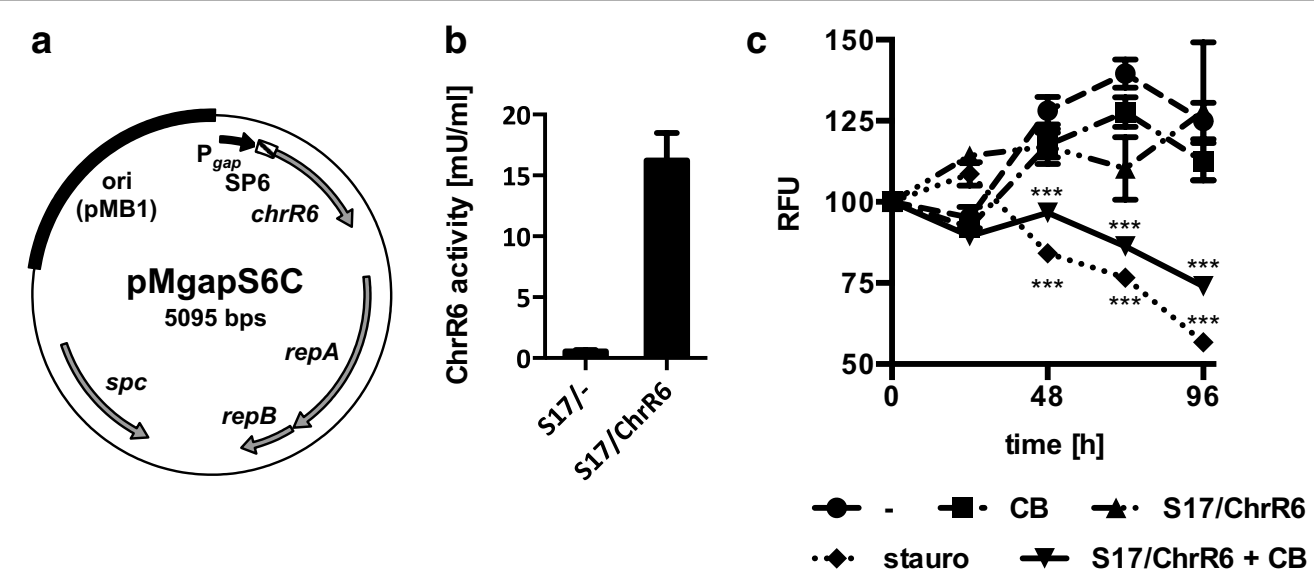

Fig. 5 Effect of B. bifidum S17/pMgapS6C and CB1954 on HT-29/EGFP4 MCTS. a Vector map of pMgapS6C for expression of a secreted chromate reductase in bifidobacteria. b Chromate reductase activity in the supernatants of B. bifidum S17/pMgapS6C (S17/ChrR6) or the empty vector control strain B. bifidum S17/pMDY23-P gap (S17/-). c HT-29/EGFP4 MCTS were incubated with S17/ChrR6 alone or in combination with $100 \mu$ M CB1954 (CB) for up to $96 \mathrm{~h}$. As controls, MCTS were treated with $200 \mathrm{nM}$ staurosporine (stauro), CB1954 (CB) or medium alone (-). Fluorescence intensities were normalized to give $100 \%$ for each MCTS at the start of the experiment $(t=0 \mathrm{~h})$. Values are relative fluorescent units (RFU) per MCTS and are mean \pm SEM of three MCTS. Results of one representative of three independent experiments are shown. Statistical analysis was performed using two-way ANOVA with Bonferroni post-test correction for multiple comparisons (***difference to untreated MCTS significant with adjusted $P<0.001$, all other comparisons not significant)

While B. bifidum S17 was detected almost exclusively in hypoxic areas of tumors, S. typhimurium YB1 also adhered to the outer surface. This might be due to differences in the labeling methods. B. bifidum S17 was detected by expression of fluorescent proteins. By contrast, S. typhimurium YB1 was labeled with a specific antibody. Whereas the former method detects only intact, metabolically active cells, antibodies stain all bacteria including dead or metabolically inactive cells. Thus, it is possible that the signal of S. typhimurium YB1 detected on the outside of MCTS represents non-replicative, inactive, or dead bacteria.

Nevertheless, S. typhimurium YB1 was shown to proliferate in MCTS at later time-points indicating that some bacteria, presumably those that reside in hypoxic areas, are viable. Similarly, expression of fluorescent proteins by B. bifidum S17 and germination of C. sporogenes spores in hypoxic areas of MCTS suggest metabolic activity. Moreover, germination of spores of strictly anaerobic $C$. sporogenes NCIMB 10696 was observed inside MCTS (Fig. 3). This suggests that oxygen levels inside MCTS are sufficiently low to support colonization of hypoxic areas of HT-29 MCTS by obligate anaerobic bacteria. Thus, the presented data confirms previous findings on selective colonization of solid tumors by these bacteria in animal models $[16,17,25,35,36]$.

These findings indicate that recombinant, tumor targeting bacteria are likely to express therapeutic genes inside MCTS. To test the potential of MCTS for studies on therapeutic approaches, derivative of the HT-29 cell line stably transfected for constitutive EGFP expression was generated. Techniques employing fluorescent labels or read-outs are widely used for high-throughput drug discovery [47, 48] and EGFP-expressing HT-29 tumor spheroids have been generated previously for the highthroughput screening of pharmacological treatments [49]. Proliferation of entire spheroids was measured by EGFP fluorescence intensity using a microtiter plate reader. This is an easy, reliable and fast measurement. While flow cytometry would be a theoretical alternative providing quantitative information on the total number of cells in spheroids and the fluorescence intensity on the single cell level, it bears downsides that are related to the spheroid model. For example, spheroids need to be disintegrated to a single cell suspension prior to flow cytometry. In fact, several attempts to reproducibly and reliably obtain single cell suspension from HT-29 MCTS by enzymatic treatment with or without mechanical disruption failed (data not shown) probably due to the tight cell-cell adhesion of cells within spheroids. Staurosporine treatment of HT-29/EGFP4 MCTS resulted in a dose-dependent inhibition of the increase in EGFP fluorescence of MCTS (Additional file 3: Figure S2C) as reported for other cell lines [50] demonstrating the principal feasibility of HT-29/EGFP4 MCTS to test therapeutic strategies.

To test therapeutic approaches using bacterial gene delivery vectors, recombinant strains of B. bifidum S17 were generated expressing two of the most widely used PCEs: cytosine deaminase and chromate reductase. Both PCEs have been used successfully in bacterial tumor 
targeting studies in animals models [51, 52]. In MCTS, a combination of B. bifidum S17/pMgapS6C and CB1954 was able to inhibit tumor growth (Fig. $5 \mathrm{c}$ ). By contrast, $B$. bifidum S17/pAO-S0_CD expressing cytosine deaminase in combination with the prodrug 5 -FC had no effect on growth of HT-29 MCTS (Fig. 4a). Further experiments revealed that the clone of HT-29 cells used for experiments had an intrinsic resistance to the active compound 5-FU produced by the PCE (Fig. 4b). Similar observations have been published for this and other colon cancer cell lines $[44,45]$.

The vast majority of reports on bacterial tumor-targeting have been performed in mouse models and there are very few studies on bacterial tumor targeting using $3 \mathrm{D}$ in vitro models. To our knowledge, there is only one study in which tumor spheroids were used to investigate the therapeutic effect of a recombinant $S$. typhimurium strain expressing a therapeutic gene [53]. A microfluidic tumor model was used to analyze proliferation of different $E$. coli strains in [54]. This system consists of a reactor chamber with a diameter $100 \mu \mathrm{m}$ connected to two outer channels perfused with oxygenated medium by pores of $4 \mu \mathrm{m}$ allowing diffusion of nutrients and oxygen. Thus, it probably does not allow for the development of extensively hypoxic areas, which in MCTS start not until approx. $100 \mu \mathrm{m}$ below the outer surface. Tumor cylindroids, which are derived from MCTS, have been used study tumor colonization by $S$. typhimurium [55-57]. Compared to MCTS, both cylindroids and microfluidics are technically by far more challenging, require significant additional equipment, and are thus not suitable for large scale or high throughput screenings.

\section{Conclusions}

Collectively, our results demonstrate that MCTS generated from HT-29 cells are a suitable model system to study tumor targeting strategies using obligate anaerobic bacteria or their spores in vitro. A wide range of other tumor cell lines have been shown to form similar MCTS when cultured appropriately [37-39]. Moreover, MCTS can be grown in a high-throughput format [42] or developed further into more complex model systems offering the opportunity to analyze the molecular crosstalk between tumor cells and other cell types frequently found in tumors such as fibroblasts or immune cells $[38,58-60]$. This may facilitate research on therapeutic approaches beyond PCE strategies, e.g. recombinant bacterial vectors expressing defined cytokines that modulate the anti-inflammatory phenotype of tumor-associated macrophages or activate infiltrating T- or NK-cells.

In conclusion, MCTS have great potential to facilitate preclinical investigation of bacterial tumor targeting. MCTS allow efficacy testing of existing bacterial vectors, the development of new therapeutic strategies involving bacterial gene delivery, or oncolytic effects of bacteria an in vitro setting before turning to time, cost, and labor intensive animal experimentation.

\section{Methods}

\section{Bacterial strains and growth conditions}

Bifidobacterial strains were grown in Lactobacilli MRS medium (Difco) supplemented with $0.5 \mathrm{~g} / \mathrm{L}$ L-cysteine (MRSc) under anaerobic conditions (AnaeroGenTM, Oxoid) at $37{ }^{\circ} \mathrm{C}$. For cultivation of B. bifidum S17 derivatives carrying plasmids pVG-mCherry, pVG-GFP, pAOS0_CD [43, 61], or pMgapS0C, MRSc was supplemented with $100 \mu \mathrm{g} / \mathrm{mL}$ spectinomycin. S. typhimurium YB1 [36] was kindly provided by Prof. Jian-Dong Huang (University of Hong Kong) and was grown aerobically at $37^{\circ} \mathrm{C}$ in DMEM cell culture medium supplemented with $50 \mu \mathrm{g} /$ $\mathrm{mL}$ DAP and $25 \mu \mathrm{g} / \mathrm{mL}$ chloramphenicol. Clostridium sporogenes NCIMB 10696 (NCIMB Ltd., Aberdeen, GB) was cultivated anaerobically in $2 \mathrm{YT}$ medium containing $3 \%(\mathrm{w} / \mathrm{v})$ tryptone, $2 \%(\mathrm{w} / \mathrm{v})$ yeast extract, and $8.7 \mathrm{mM}$ sodium thioglycolate.

For generation of C. sporogenes spores, $50 \mathrm{~mL}$ medium was inoculated and incubated for 2 weeks at $30{ }^{\circ} \mathrm{C}$. Sporulation was monitored microscopically. Spores were harvested by centrifugation $\left(30 \mathrm{~min}, 4{ }^{\circ} \mathrm{C}, 4500 \times g\right)$ and incubated for $30 \mathrm{~min}$ in $50 \mathrm{~mL} 80 \%$ (v/v) ethanol to kill any remaining vegetative cells. The pellet was washed three times with $50 \mathrm{~mL}$ phosphate buffered solution (PBS) and suspended in $125 \mu \mathrm{L}$ PBS. Spores were separated by density centrifugation using Percoll ${ }^{\circledR}$. For this purpose $900 \mu \mathrm{L}$ of filtrated $1.5 \mathrm{M} \mathrm{NaCl}$ was added to $8.1 \mathrm{~mL}$ of Percoll $^{\circledR}$ solution (Sigma-Aldrich Chemie $\mathrm{GmbH}$, Munich, Germany). $9 \mathrm{~mL}$ of this stock solution were mixed with $1 \mathrm{~mL}$ of $0.15 \mathrm{M} \mathrm{NaCl}$. A gradient was prepared by centrifugation using a swinging bucket rotor $(1 \mathrm{~h}$, room temperature, $4500 \times g) .125 \mu \mathrm{L}$ of crude spore preparation was applied on top of the gradient followed by centrifugation $(1 \mathrm{~h}$, room temperature, $1800 \times g)$. The upper $9 \mathrm{~mL}$ of the Percoll ${ }^{\circledR} / \mathrm{NaCl}$ gradient were removed and the spore sediment was resuspended in $5 \mathrm{~mL}$ of PBS followed by centrifugation $(5 \mathrm{~min}, \mathrm{RT}, 9600 \times \mathrm{g}$ ). The pellet was washed three times with PBS and resuspended in $1 \mathrm{~mL}$ PBS. Absence of vegetative cells was checked microscopically. The concentration of colony-forming units (CFU) of the spore preparation was determined by plating on $2 \mathrm{YT}$ agar and cultivation at $30{ }^{\circ} \mathrm{C}$ under anaerobic conditions.

\section{Cell culture and generation of solid 3D MCTS in vitro}

HT-29 colorectal adenocarcinoma cells (ATCC ${ }^{\circledR}$ HTB$38^{\mathrm{TM}}$ ) were routinely cultured in DMEM medium supplemented with $5 \%(\mathrm{v} / \mathrm{v})$ FCS, $1 \%(\mathrm{v} / \mathrm{v})$ non-essential 
amino acids, and $1 \%(\mathrm{v} / \mathrm{v})$ penicillin-streptomycin stock solution at $37^{\circ} \mathrm{C}$ with $5 \% \mathrm{CO}_{2}$. For generation of MCTS, cells were seeded in Ultra-Low Attachment 96-well plates $\left(\right.$ Corning $^{\circledR} \operatorname{Costar}^{\circledR}$ ) at $1 \times 10^{5}$ cells in $200 \mu \mathrm{L}$ medium per well. Cells were grown for 14 days with medium changed every 2-3 days. MCTS with a diameter of 500$1000 \mu \mathrm{m}$ were used for experiments.

To generate green fluorescent MCTS, HT-29 cells were transfected with pEGFP-N3 (Clontech Laboratories, Inc., USA) and a stable clone HT-29/EGFP4 was selected as described in Additional file 2: Materials S2. HT-29/ EGFP4 MCTS were generated using DMEM medium without phenol red to avoid interference with EGFP fluorescence measurements. Growth of HT-29/EGFP4 MCTS was monitored by quantification of EGFP fluorescence as an indirect measurement of cell proliferation and MCTS size. Fluorescence intensity of HT-29/EGFP4 MCTS was measured directly in tissue culture plates using a Tecan Infinite ${ }^{\circledR}$ M200 multimode reader (Tecan Group Ltd., Mannedorf, Switzerland) with excitation at $477 \mathrm{~nm}$ and emission at $507 \mathrm{~nm}$.

\section{Bacterial tumor targeting assays}

For survival studies in MCTS, bifidobacteria were inoculated in $10 \mathrm{~mL}$ MRSc supplemented with antibiotics as appropriate. S. typhimurium YB1 was inoculated in $10 \mathrm{~mL}$ DMEM containing $50 \mu \mathrm{g} / \mathrm{mL}$ DAP and $25 \mu \mathrm{g} /$ $\mathrm{mL}$ chloramphenicol. Bacteria were grown anaerobically (bifidobacteria) or aerobically (S. typhimurium YB1) at $37^{\circ} \mathrm{C}$ over night (o/N; i.e. for $16 \mathrm{~h}$ ) to stationary growth phase. At this stage, an optical density at $600 \mathrm{~nm}$ $\left(\mathrm{OD}_{600}\right)$ of 1 equals approximately $1 \times 10^{8}$ (bifidobacteria) or $1 \times 10^{9}$ (S. typhimurium YB1) CFU/mL. Bacteria were washed once and resuspended in PBS to a final $\mathrm{OD}_{600}$ of 0.1 (bifidobacteria) or 0.01 (S. typhimurium YB1). The $C$. sporogenes spore preparation was adjusted to $5 \times 10^{5} \mathrm{CFU} / \mathrm{mL}$.

Prior to inoculation, single MCTS in individual wells were washed once in DMEM and $180 \mu \mathrm{L}$ of DMEM supplemented with $60 \mathrm{mM}$ HEPES buffer (without FCS or antibiotics) were added. Aliquots of $20 \mu \mathrm{L}$ of the bacterial suspensions or spore stocks were added to individual wells resulting in an inoculum of $2 \times 10^{7} \mathrm{CFU}$ or $1 \times 10^{3}$ spores per MCTS. At the indicated time-points after infection, six MCTS infected with the same strain were combined. Homogenous suspensions of tumor cells were prepared by passing through a syringe $(0.4 \mathrm{~mm}) 5$ times with vortexing between homogenization steps. Bacterial numbers were determined as $\mathrm{CFU} / \mathrm{mL}$ in the MCTS lysates by plating serial ten-fold dilutions in PBS on agar plates (bifidobacteria: MRSc agar; S. typhimurium YB1: LB agar containing $50 \mu \mathrm{g} / \mathrm{mL}$ DAP after growth under standard conditions. For assays with $C$. sporogenes spores, MCTS were infected and incubated for 7 days under cell culture conditions. At this stage tumors were examined by fluorescence microscopy.

For experiments with recombinant bifidobacteria expressing PCEs, MCTS were incubated with $2 \times 10^{7}$ CFU. Medium was changed after $72 \mathrm{~h}$ of incubation and fresh prodrug was added to MCTS.

\section{Microscopic examination of MCTS}

Individual MCTS incubated with bacteria or spores for the indicated time were embedded in a drop of TissueTek ${ }^{\circledR}$ O.C.T Compound (Sakura) in a Leica CM3050S cryostat and immediately used to prepare cryosections (thickness 10-14 $\mu \mathrm{m}$ ), which were then dried on Superfrost ${ }^{\mathrm{TM}}$ Plus microscope slides (Fisher Scientific) and fixed with $4 \%$ paraformaldehyde/PBS.

Fixed MCTS were stained with hematoxylin/eosin (H\&E). Hoechst (Life Technologies) was used at $300 \mathrm{nM}$ to stain cell nuclei and cytoskeletal actin was stained using Alexa Fluor ${ }^{\circledR} 488$ Phalloidin (Invitrogen). Hypoxyprobe-Red549 Kit (Hypoxyprobe) was used to stain for hypoxic areas and apoptotic cells were stained by TUNEL assay (DNA Fragmentation Imaging Kit, Roche). Proliferating cells were visualized by Ki-67 staining using a mouse monoclonal antibody (Cat\# MA1-80199, Thermo Scientific) and goat $\alpha$-mouse IgG Alexa Fluor ${ }^{\circledR} 555$ secondary antibody (Life Technologies). S. typhimurium YB1 was stained with a rabbit polyclonal Salmonella antiserum (Cat\# PA1-7244, Thermo Scientific) and goat $\alpha$-rabbit Alexa Fluor ${ }^{\circledR} 488$ or 555 secondary antibodies (Life Technologies). Clostridial spores were stained with polyclonal, FITC-conjugated Clostridia species antibodies (Cat\# PA1-73169, Thermo Scientific). In a mix of vegetative $C$. sporogenes cells only spores were stained by the antibody while DNA of vegetative cells was stained by Hoechst demonstrating specificity of the antibody for spores.

Microscopic analysis was performed with an Axio Observer.Z1 microscope (Zeiss) using Zen software. The following filter sets were used for fluorescence microscopy: 49 (excitation at $365 \pm 10 \mathrm{~nm}$, emission at $445 \pm 50 \mathrm{~nm}$ ) for Hoechst, 38HE (excitation at $470 \pm 40 \mathrm{~nm}$, emission at $525 \pm 50 \mathrm{~nm}$ ) for GFP, TUNEL, FITC and Alexa Fluor ${ }^{\circledR} 488$, and AF (excitation at $545 \pm 30 \mathrm{~nm}$, emission at $610 \pm 75 \mathrm{~nm}$ ) for mCherry, Hypoxyprobe-Red549, and Alexa Fluor ${ }^{\circledR} 555$.

\section{Cloning of PCE-expressing B. bifidum S17 strains}

Bifidobacterium bifidum S17/pAO-S0_CD expressing a secreted cytosine deaminase has been described previously [43]. A similar construct was devised to generate a recombinant B. bifidum S17 strain expressing a secreted chromate reductase. For this purpose, the chrR6 gene 
encoding an artificial nitroreductase created by mutation of the $E$. coli yieF gene, which shows faster enzyme kinetics than its native form, was used [62]. The chrR6 gene was codon-optimized for B. bifidum S17 and fused in silico to the coding sequence of $\mathrm{S} 6$, a signal peptide of $B$. bifidum S17 for efficient protein secretion [43]. The codon-optimised fusion was synthesised by a commercial provider (Eurofins Genomics GmbH; Germany) and amplified by PCR using primers S6F (3'-GGCCTCGAGATGAAATCA CTGATGAAAAAGGTTTTCGC-5') and ChrR (3'-CCC AAGCTTTTAAATTTTCACGCGTTG-5') to introduce appropriate restriction sites (underlined in primer sequences). The obtained PCR product was digested with restriction enzymes XhoI and HindIII and ligated to the 4423 bp fragment of Xhol/HindIII digested pMDY23$\mathrm{P}_{\text {gap }}$ [63] yielding pMgapS6C, which encodes an exact transcriptional fusion of the S6-chrR6 sequence to the gap promoter of B. bifidum S17 for strong constitutive expression [64].

Following transformation into $E$. coli, plasmids of spectinomycin resistant colonies were checked for correct inserts by PCR. Plasmids of positive clones were verified by restriction analysis and Sanger sequencing. A plasmid with correct sequences and a mutation-free insert was termed pMgapS6C. This plasmid and the empty vector control (pMDY23- $\mathrm{P}_{\text {gap }}$ ) were transformed into B. bifidum S17 as described elsewhere [65]. Chromate reductase activity was measured in culture supernatants of recombinant bifidobacteria grown for $16 \mathrm{~h}$ in Reinforced Clostridium Medium at $37^{\circ} \mathrm{C}$ under anaerobic conditions using a previously described enzymatic assay [66].

\section{Additional files}

Additional file 1: Figure S1. Characterisation of HT-29 MCTS.

Additional file 2: Materials S2. Generation of HT-29/GFP4 cells.

Additional file 3: Figure S2. Characterization of HT-29 and HT-29/EGFP4 cells.

\section{Abbreviations}

3D: three-dimensional; MCTS: multicellular tumor spheroids; PCE: prodrugconverting enzyme; 5-FC: 5-fluorocytosine; 5-FU: 5-fluorouracil.

\section{Authors' contributions}

AO, ZS, AMW, WS, PD, and CUR conceived the study, AO, VG, GA, and KR carried out in vitro experiments. $\mathrm{AO}$ and $\mathrm{ZS}$ generated plasmids and recombinant strains. AO, ZS, and CUR analyzed the data. AO and CUR CR drafted the manuscript and all the authors contributed to preparing the final version of the manuscript. All authors read and approved the final manuscript.

\section{Author details}

${ }^{1}$ Institute of Microbiology and Biotechnology, University of Ulm, 89069 UIm, Germany. ${ }^{2}$ College of Life Sciences and Agriculture, Zhoukou Normal University, Chuanhui District, Zhoukou 466001, People's Republic of China. ${ }^{3}$ Institute of Medical Microbiology, University Hospital Essen, University Duisburg-Essen, Essen, Germany. ${ }^{4}$ Department of Lead Discovery, Boehringer Ingelheim RCV
GmbH \& Co KG, 1121 Vienna, Austria. ${ }^{5}$ Institute of Applied Biotechnology, University of Applied Sciences Biberach, 88400 Biberach, Germany.

\section{Acknowledgements}

This study was partially funded by the German Academic Exchange Service and Federal Ministry of Education and Research (Grant D/09/04778 to CUR) $\mathrm{AO}$ and KR were supported by PhD fellowships of the "Landesgraduiertenförderung Baden-Württemberg", within the "Kooperatives Promotionskolleg Pharmazeutische Biotechnologie der Universität Ulm und der Hochschule Biberach", funded by the Ministry of Science and Arts of Baden-Württemberg, and $\mathrm{AO}$ is part of the International Graduate School of Molecular Medicine at the University of Ulm. ZS received a PhD fellowship of the German Academic Exchange Service. The authors gratefully acknowledge Prof. Jian-Dong Huang (University of Hong Kong) for generously providing S. typhimurium YB1.

\section{Competing interests}

The authors declare that they have no competing interests.

Received: 16 September 2015 Accepted: 11 November 2015 Published online: 12 December 2015

\section{References}

1. Egeblad M, Nakasone ES, Werb Z. Tumors as organs: complex tissues that interface with the entire organism. Dev Cell. 2010;18:884-901.

2. Vaupel P, Kallinowski F, Okunieff P. Blood flow, oxygen and nutrient supply, and metabolic microenvironment of human tumors: a review. Cancer Res. 1989;49:6449-65.

3. Elliott NT, Yuan F. A review of three-dimensional in vitro tissue models for drug discovery and transport studies. J Pharm Sci. 2011;100:59-74.

4. Shannon AM, Bouchier-Hayes DJ, Condron CM, Toomey D. Tumour hypoxia, chemotherapeutic resistance and hypoxia-related therapies. Cancer Treat Rev. 2003;29:297-307

5. Trédan O, Galmarini CM, Patel K, Tannock IF. Drug resistance and the solid tumor microenvironment. J Natl Cancer Inst. 2007;99:1441-54.

6. Brown JM, Wilson WR. Exploiting tumour hypoxia in cancer treatment. Nat Rev Cancer. 2004;4:437-47.

7. Minchinton Al, Tannock IF. Drug penetration in solid tumours. Nat Rev Cancer. 2006;6:583-92.

8. Jiang S-N, Phan TX, Nam T-K, Nguyen VH, Kim H-S, Bom H-S, Choy HE, Hong Y, Min J-J. Inhibition of tumor growth and metastasis by a combination of Escherichia coli-mediated cytolytic therapy and radiotherapy. Mol Ther J Am Soc Gene Ther. 2010;18:635-42.

9. Weibel S, Stritzker J, Eck M, Goebel W, Szalay AA. Colonization of experimental murine breast tumours by Escherichia coli K-12 significantly alters the tumour microenvironment. Cell Microbiol. 2008;10:1235-48.

10. Riedel CU, Monk IR, Casey PG, Morrissey D, O'Sullivan GC, Tangney M, Hill C, Gahan CGM. Improved luciferase tagging system for Listeria monocytogenes allows real-time monitoring in vivo and in vitro. Appl Environ Microbiol. 2007:73:3091-4.

11. Quispe-Tintaya W, Chandra D, Jahangir A, Harris M, Casadevall A, Dadachova E, Gravekamp C. Nontoxic radioactive Listeria(at) is a highly effective therapy against metastatic pancreatic cancer. Proc Natl Acad Sci USA. 2013;110:8668-73.

12. Baban CK, Cronin M, O'Hanlon D, O'Sullivan GC, Tangney M. Bacteria as vectors for gene therapy of cancer. Bioeng Bugs. 2010;1:385-94.

13. Forbes NS. Engineering the perfect (bacterial) cancer therapy. Nat Rev Cancer. 2010;10:785-94.

14. Pawelek JM, Low KB, Bermudes D. Bacteria as tumour-targeting vectors. Lancet Oncol. 2003:4:548-56.

15. Taniguchi S, Fujimori M, Sasaki T, Tsutsui H, Shimatani Y, Seki K, Amano J. Targeting solid tumors with non-pathogenic obligate anaerobic bacteria. Cancer Sci. 2010;101:1925-32.

16. Cronin M, Morrissey D, Rajendran S, El Mashad SM, van Sinderen D, O'Sullivan GC, Tangney M. Orally administered bifidobacteria as vehicles for delivery of agents to systemic tumors. Mol Ther. 2010;18:1397-407.

17. Cronin M, Akin AR, Collins SA, Meganck J, Kim J-B, Baban CK, Joyce SA, van Dam GM, Zhang N, van Sinderen D, O'Sullivan GC, Kasahara N, Gahan CG, Francis KP, Tangney M. High resolution in vivo bioluminescent 
imaging for the study of bacterial tumour targeting. PLoS One. 2012;7:e30940.

18. Hu B, Kou L, Li C, Zhu L-P, Fan Y-R, Wu Z-W, Wang J-J, Xu G-X. Bifidobacterium longum as a delivery system of TRAIL and endostatin cooperates with chemotherapeutic drugs to inhibit hypoxic tumor growth. Cancer Gene Ther. 2009;16:655-63.

19. Hidaka A, Hamaji Y, Sasaki T, Taniguchi S, Fujimori M. Exogenous cytosine deaminase gene expression in Bifidobacterium breve I-53-8w for tumor-targeting enzyme/prodrug therapy. Biosci Biotechnol Biochem. 2007;71:2921-6.

20. Tang W, He Y, Zhou S, Ma Y, Liu G. A novel Bifidobacterium infantis-mediated TK/GCV suicide gene therapy system exhibits antitumor activity in a rat model of bladder cancer. J Exp Clin Cancer Res CR. 2009;28:155.

21. Van Mellaert L, Barbé $S$, Anné J. Clostridium spores as anti-tumour agents. Trends Microbiol. 2006;14:190-6.

22. Minton NP. Clostridia in cancer therapy. Nat Rev Microbiol. 2003;1:237-42.

23. Liu SC, Minton NP, Giaccia AJ, Brown JM. Anticancer efficacy of systemically delivered anaerobic bacteria as gene therapy vectors targeting tumor hypoxia/necrosis. Gene Ther. 2002;9:291-6.

24. Wei MQ, Mengesha A, Good D, Anné J. Bacterial targeted tumour therapy-dawn of a new era. Cancer Lett. 2008;259:16-27.

25. Dang LH, Bettegowda C, Huso DL, Kinzler KW, Vogelstein B. Combination bacteriolytic therapy for the treatment of experimental tumors. Proc Natl Acad Sci USA. 2001;98:15155-60.

26. Malmgren RA, Flanigan CC. Localization of the vegetative form of Clostridium tetani in mouse tumors following intravenous spore administration. Cancer Res. 1955;15:473-8.

27. Safety study of Clostridium novyi-NT spores to treat patients with solid tumors that have not responded to standard therapies. https://www. clinicaltrials.gov/ct2/show/record/NCT01118819.

28. Diaz LA, Cheong I, Foss CA, Zhang X, Peters BA, Agrawal N, Bettegowda C, Karim B, Liu G, Khan K, Huang X, Kohli M, Dang LH, Hwang P, Vogelstein A, Garrett-Mayer E, Kobrin B, Pomper M, Zhou S, Kinzler KW, Vogelstein B, Huso DL. Pharmacologic and toxicologic evaluation of C. novyi-NT spores. Toxicol Sci. 2005;88:562-75.

29. Leschner S, Weiss S. Salmonella-allies in the fight against cancer. J Mol Med (Berl). 2010;88:763-73.

30. Chorobik P, Czaplicki D, Ossysek K, Bereta J. Salmonella and cancer: from pathogens to therapeutics. Acta Biochim Pol. 2013;60:285-97.

31. Broadway KM, Modise T, Jensen RV, Scharf BE. Complete genome sequence of Salmonella enterica serovar Typhimurium VNP20009, a strain engineered for tumor targeting. J Biotechnol. 2014;192 Pt A:177-8.

32. Low KB, Ittensohn M, Le T, Platt J, Sodi S, Amoss M, Ash O, Carmichael E, Chakraborty A, Fischer J, Lin SL, Luo X, Miller SI, Zheng L, King I, Pawelek JM, Bermudes D. Lipid A mutant Salmonella with suppressed virulence and TNFalpha induction retain tumor-targeting in vivo. Nat Biotechnol. 1999;17:37-41.

33. Hoiseth SK, Stocker BA. Aromatic-dependent Salmonella typhimurium are non-virulent and effective as live vaccines. Nature. 1981;291:238-9.

34. Zhao M, Yang M, Ma H, Li X, Tan X, Li S, Yang Z, Hoffman RM. Targeted therapy with a Salmonella typhimurium leucine-arginine auxotroph cures orthotopic human breast tumors in nude mice. Cancer Res. 2006;66:7647-52.

35. Clairmont C, Lee KC, Pike J, Ittensohn M, Low KB, Pawelek J, Bermudes D, Brecher SM, Margitich D, Turnier J, Li Z, Luo X, King I, Zheng LM. Biodistribution and genetic stability of the novel antitumor agent VNP20009, a genetically modified strain of Salmonella typhimurium. J Infect Dis. 2000;181:1996-2002.

36. Yu B, Yang M, Shi L, Yao Y, Jiang $Q$, Li X, Tang L-H, Zheng B-J, Yuen K-Y, Smith DK, Song E, Huang J-D. Explicit hypoxia targeting with tumor suppression by creating an "obligate" anaerobic Salmonella typhimurium strain. Sci Rep. 2012;2:436.

37. Friedrich J, Ebner R, Kunz-Schughart LA. Experimental anti-tumor therapy in 3-D: spheroids_old hat or new challenge? Int J Radiat Biol. 2007;83:849-71

38. Friedrich J, Seidel C, Ebner R, Kunz-Schughart LA. Spheroid-based drug screen: considerations and practical approach. Nat Protoc. 2009;4:309-24.

39. Hirschhaeuser F, Menne H, Dittfeld C, West J, Mueller-Klieser W, KunzSchughart LA. Multicellular tumor spheroids: an underestimated tool is catching up again. J Biotechnol. 2010;148:3-15.
40. L'Espérance S, Bachvarova M, Tetu B, Mes-Masson A-M, Bachvarov D. Global gene expression analysis of early response to chemotherapy treatment in ovarian cancer spheroids. BMC Genomics. 2008;9:99.

41. Zietarska M, Maugard CM, Filali-Mouhim A, Alam-Fahmy M, Tonin PN, Provencher DM, Mes-Masson A-M. Molecular description of a 3D in vitro model for the study of epithelial ovarian cancer (EOC). Mol Carcinog. 2007:46:872-85.

42. Gong X, Lin C, Cheng J, Su J, Zhao H, Liu T, Wen X, Zhao P. Generation of multicellular tumor spheroids with microwell-based agarose scaffolds for drug testing. PLoS One. 2015;10:e0130348.

43. Osswald A, Westermann C, Sun Z, Riedel CU. A phytase-based reporter system for identification of functional secretion signals in bifidobacteria. PLoS One. 2015;10:e0128802.

44. Violette S, Poulain L, Dussaulx E, Pepin D, Faussat A-M, Chambaz J, Lacorte J-M, Staedel C, Lesuffleur T. Resistance of colon cancer cells to long-term 5-fluorouracil exposure is correlated to the relative level of $\mathrm{BCl}-2$ and $\mathrm{BCl}-$ $X(L)$ in addition to Bax and p53 status. Int J Cancer. 2002;98:498-504.

45. Leteurtre E, Gouyer V, Rousseau K, Moreau O, Barbat A, Swallow D, Huet $\mathrm{G}$, Lesuffleur T. Differential mucin expression in colon carcinoma HT-29 clones with variable resistance to 5 -fluorouracil and methotrexate. Bio Cell. 2004;96:145-51.

46. Green NK, Kerr DJ, Mautner V, Harris PA, Searle PF. The nitroreductase/ CB1954 enzyme-prodrug system. Methods Mol Med. 2004;90:459-77.

47. Eggeling C, Brand L, Ullmann D, Jäger S. Highly sensitive fluorescence detection technology currently available for HTS. Drug Discov Today. 2003:8:632-41.

48. Gribbon P, Sewing A. Fluorescence readouts in HTS: no gain without pain? Drug Discov Today. 2003;8:1035-43.

49. Zhang X, Yang S-T. High-throughput 3-D cell-based proliferation and cytotoxicity assays for drug screening and bioprocess development. J Biotechnol. 2011;151:186-93.

50. Steff AM, Fortin M, Arguin C, Hugo P. Detection of a decrease in green fluorescent protein fluorescence for the monitoring of cell death: an assay amenable to high-throughput screening technologies. Cytometry. 2001;45:237-43

51. Lehouritis P, Springer C, Tangney M. Bacterial-directed enzyme prodrug therapy. J Control Release. 2013;170:120-31.

52. Zu C, Wang J. Tumor-colonizing bacteria: a potential tumor targeting therapy. Crit Rev Microbiol. 2014;40:225-35.

53. Ryan RM, Green J, Williams PJ, Tazzyman S, Hunt S, Harmey JH, Kehoe SC, Lewis CE. Bacterial delivery of a novel cytolysin to hypoxic areas of solid tumors. Gene Ther. 2009;16:329-39.

54. Elliott N, Lee T, You L, Yuan F. Proliferation behavior of E. coli in a threedimensional in vitro tumor model. Integr Biol Quant. 2011;3:696-705.

55. Forbes NS, Munn LL, Fukumura D, Jain RK. Sparse initial entrapment of systemically injected Salmonella typhimurium leads to heterogeneous accumulation within tumors. Cancer Res. 2003;63:5188-93.

56. Kasinskas RW, Forbes NS. Salmonella typhimurium specifically chemotax and proliferate in heterogeneous tumor tissue in vitro. Biotechnol Bioeng. 2006;94:710-21

57. Kasinskas RW, Forbes NS. Salmonella typhimurium lacking ribose chemoreceptors localize in tumor quiescence and induce apoptosis. Cancer Res. 2007:67:3201-9.

58. Furbert-Harris PM, Hunter KA, Vaughn TR, Parish-Gause D, Laniyan I, Harris D, Okomo-Awich J, Forrest K, Oredipe OA. Eosinophils in a tri-cell multicellular tumor spheroid (MTS)/endothelium complex. Cell Mol Biol (Noisy-le-grand). 2003;49:1081-8.

59. Gottfried E, Kunz-Schughart LA, Andreesen R, Kreutz M. Brave little world: spheroids as an in vitro model to study tumor-immune-cell interactions. Cell Cycle. 2006:5:691-5.

60. Rudisch A, Dewhurst MR, Horga LG, Kramer N, Harrer N, Dong M, van der Kuip H, Wernitznig A, Bernthaler A, Dolznig H, Sommergruber W. High EMT signature score of invasive non-small cell lung cancer (NSCLC) cells correlates with NFKB driven colony-stimulating factor 2 (CSF2/GM-CSF) secretion by neighboring stromal fibroblasts. PLoS One. 2015;10:e0124283.

61. Grimm V, Gleinser M, Neu C, Zhurina D, Riedel CU. Expression of fluorescent proteins in bifidobacteria for analysis of host-microbe interactions. Appl Environ Microbiol. 2014;80:2842-50. 
62. Thorne SH, Barak Y, Liang W, Bachmann MH, Rao J, Contag CH, Matin A. CNOB/ChrR6, a new prodrug enzyme cancer chemotherapy. Mol Cancer Ther. 2009;8:333-41.

63. Gleinser M, Grimm V, Zhurina D, Yuan J, Riedel CU. Improved adhesive properties of recombinant bifidobacteria expressing the Bifidobacterium bifidum-specific lipoprotein BopA. Microb Cell Fact. 2012;11:80.

64. Sun Z, Westermann C, Yuan J, Riedel CU. Experimental determination and characterization of the gap promoter of Bifidobacterium bifidum S17. Bioengineered. 2014;55(6):371-7.
65. MacConaill LE, Fitzgerald GF, Van Sinderen D. Investigation of protein export in Bifidobacterium breve UCC2003. Appl Environ Microbiol. 2003;69:6994-7001.

66. Race PR, Lovering AL, White SA, Grove Jl, Searle PF, Wrighton CW, Hyde El. Kinetic and structural characterisation of Escherichia coli nitroreductase mutants showing improved efficacy for the prodrug substrate CB1954. J Mol Biol. 2007;368:481-92.
Submit your next manuscript to BioMed Central and we will help you at every step:

- We accept pre-submission inquiries

- Our selector tool helps you to find the most relevant journal

- We provide round the clock customer support

- Convenient online submission

- Thorough peer review

- Inclusion in PubMed and all major indexing services

- Maximum visibility for your research

Submit your manuscript at www.biomedcentral.com/submit
() Biomed Central 